\title{
Lu pour Vous
}

\section{Génétique}

Tuy Nga Brignol

\section{Apport du séquençage de nouvelle génération (NGS) dans le diagnostic des maladies neuromusculaires}

\section{Résumé}

Cet article de revue d'عfthymiou et al. [1] fait partie d'un numéro spécial d'octobre 2016 de Current Opinion in Neurology sur les maladies neuromusculaires [2]. Les gènes des MNM, souvent de grande taille, contiennent de nombreux polymorphismes, rendant difficile l'identification des variants pathogènes. Cela est particulièrement vrai pour les gènes $D M D$ de la dystrophine $(>2,3$ Mb, 79 exons), TTN de la titine (> $100 \mathrm{~kb}, 363$ exons) et $N E B$ de la nébuline (183 exons). Dans le contexte d'une histoire familiale où frères et sœurs sont atteints, le diagnostic d'une MNM est en général évident. Toutefois, le mode de transmission reste souvent difficile à définir dans des petites familles où l'histoire de la famille fait défaut, ou chez des cas apparemment «sporadiques » pouvant prendre n'importe quel mode de transmission ou être de novo dominant.

Vignette (๑ Wikipedia, By Abizar at en.wikipedia, CC BY-SA 3.0, - https://commons. wikimedia.org/w/index.php?curid=3800855)

https://en.wikipedia.org/wiki/DNA_sequencing\#/media/File:Radioactive_Fluorescent_Seq.jpg

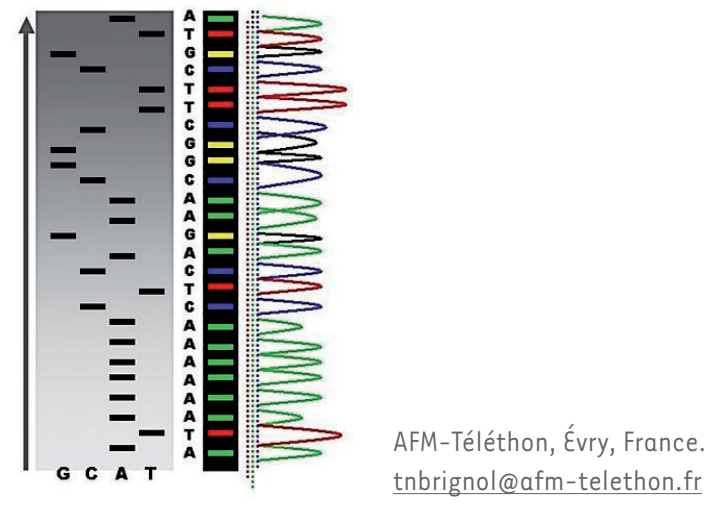

L'utilisation du NGS permet de résoudre de plus en plus des cas difficiles mais l'analyse des données est loin d'être simple. L'absence d'histoire familiale en présence de phénotypes MNM peu spécifiques complexifie l'interprétation des données du NGS, nécessitant souvent l'étude d'autres membres sains de la famille et la poursuite d'analyses fonctionnelles. Une analyse clinique de qualité ainsi qu'une bonne collaboration entre généticiens et cliniciens sont essentiels pour parvenir à une interprétation correcte des résultats.

Cependant, le taux d'erreur avec le NGS reste élevé par rapport aux méthodes classiques de séquençage (type Sanger). Des mutations peuvent être artificiellement produites lors de l'amplification, source de résultats faussement positifs. Un autre défi est lié à la détection des répétitions d'expansions ou de variations structurelles. Ces mutations peuvent passer inaperçues si elles sont supérieures à une longueur de lecture de 150 paires de base, conduisant à des résultats faussement négatifs. Par ailleurs, parmi les nombreux variants détectés par le NGS, il est souvent difficile de faire la distinction entre les mutations authentiquement pathogènes et les variations individuelles rares sans signification clinique.

Une autre difficulté est la couverture incomplète des bibliothèques de capture disponibles dans le commerce. Bien que de nouvelles versions des kits de capture soient proposées de façon régulière, aucune capture de toutes les parties codantes de tous les gènes n'est actuellement disponible. Ceci peut être résolu par le séquençage ciblé de régions d'intérêt et par l'amélioration du processus de capture. L'augmentation de la couverture de la séquence, une longueur de lecture supérieure à 150 pb, l'amélioration des algorithmes vont également permettre de résoudre certaines limites du NGS. Une autre alternative est la combinaison des différentes méthodes de séquençage.

\section{Commentaire}

Les maladies neuromusculaires sont d'une grande hétérogénéité clinique et génétique. Par exemple, pour les MNM à début précoce 
telles que les myopathies congénitales, les dystrophies musculaires congénitales et les syndromes myasthéniques congénitaux, il existe une grande hétérogénéité, avec une extension sans précédent des « diagnostics par séquençage » suite au NGS ainsi que des corrélations phénotype-génotype [3]. Plus que jamais, des évaluations cliniques, incluant l'imagerie musculaire par RMN, sont nécessaires pour guider le diagnostic génétique moléculaire. II est maintenant techniquement, cliniquement et éthiquement possible d'effectuer le NGS pour des pathologies graves à l'échelle de la population; c'est notamment le cas pour le dépistage des porteurs avant un projet parental, ou de dépistage néonatal de certaines MNM à début précoce.

Un pourcentage significatif de patients reste cependant sans diagnostic moléculaire, ce qui suggère que beaucoup de gènes et de mécanismes seraient encore à identifier. De nombreux variants «pathogènes » souvent rapportés dans les populations témoins seraient plutôt des polymorphismes rares. La validation fonctionnelle des candidats variants d'une pathologie est cruciale pour une interprétation précise du séquençage NGS et pour un conseil génétique approprié. La recherche de polymorphismes et de mutations dans un gène particulier au niveau international par les centres de référence est devenue une nécessité de plus en plus évidente, et la dissémination et la mise en commun de ces informations est importante.
Malgré certaines limites, le NGS a considérablement fait progresser la recherche et la pratique du diagnostic génétique de routine dans les MNM. Son coût ne cesse de diminuer et de nouvelles améliorations techniques sont attendues dans les prochaines années. Elles vont permettre d'obtenir plus rapidement des données encore plus précises et fiables. Si actuellement, l'étape limitante reste la validation fonctionnelle des nouveaux gènes/variants identifiés, le NGS devrait se généraliser afin de déterminer dans tous les cas la nature de la mutation causale, dont la connaissance est indispensable pour un diagnostic correct et pour l'inclusion dans les essais thérapeutiques. $\diamond$

Added value of next generation sequencing (NGS) in the diagnosis of neuromuscular disorders

\section{LIENS D'INTÉRÊT}

L'auteur déclare n'avoir aucun lien d'intérêt concernant les données publiées dans cet article.

\section{RÉFÉRENCES}

1. Efthymiou S, Manole A, Houlden H. Next-generation sequencing in neuromuscular diseases. Curr Opin Neurol 2016 ; 29 : 527-36.

2. http://journals.Iww.com/co-neurology/pages/currenttoc.aspx

3. Ravenscroft $G$, Davis MR, Lamont $P$, et al. New era in genetics of early-onset muscle disease: breakthroughs and challenges. Semin Cell Dev Biol 2016 ; pii : S1084-9521(16)30241-5.

\section{Et si $100 \%$ des reçus étaient abonnés à médecine/sciences?
Une seule solution Abonnez-vous d̀ $\mathrm{m} / \mathrm{s}$}

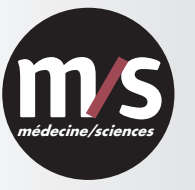

www.medecinesciences.org

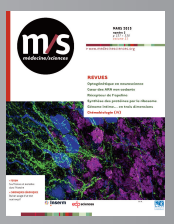

Nom - Prénom :

Institution :

Adresse :

CP

Localité :

Pays

Tél. :

E-mail (obligatoire) :

\begin{tabular}{|c|c|c|c|c|c|c|c|c|}
\hline \multirow{2}{*}{$\begin{array}{l}\text { PRIX } \\
\text { TVA 2,1\% }\end{array}$} & \multicolumn{2}{|c|}{ Institutions } & \multicolumn{2}{|c|}{ Individuels } & \multicolumn{2}{|c|}{ Étudiants* } & \multicolumn{2}{|c|}{ Enseignants* } \\
\hline & $P+E$ & $E$ & $P+E$ & $E$ & $P+E$ & $E$ & $P+E$ & $E$ \\
\hline France & $\begin{array}{l}528 € H T \\
539 € T T C\end{array}$ & \multirow{3}{*}{$\begin{array}{c}\text { Nous } \\
\text { contacter }\end{array}$} & O $230 € T T C$ & \multirow{2}{*}{ O $137 €$ TTC } & $\mathrm{O} 122 € \mathrm{TTC}$ & \multirow{2}{*}{$\mathrm{O} 81 € \mathrm{TTC}$} & O $152 € \pi C$ & \multirow{2}{*}{ O $108 €$ TTC } \\
\hline $\begin{array}{l}\text { Reste de } \\
\text { I'U.E. }\end{array}$ & $\begin{array}{l}\quad 644 € H T \\
\quad 657 € T T C\end{array}$ & & $312 € \mathrm{TTC}$ & & O $172 € \mathrm{TTC}$ & & O $262 € \pi T C$ & \\
\hline $\begin{array}{l}\text { Reste du } \\
\text { Monde }\end{array}$ & $0668 €$ & & $O 312 €$ & $O 135 €$ & O $194 €$ & O $79 €$ & $0282 €$ & O $107 €$ \\
\hline
\end{tabular}

Paiement $\square$ Envoyez-moi une facture proforma

$\mathrm{P}+\mathrm{E}$ : Papier et Électronique / E : Électronique seule

$\square$ Chèque joint (à l'ordre d'EDP Sciences)

$\square$ Carte de crédit : $\square$ Visa $\square$ Eurocard $\square$ American Express

$N^{\circ}$ de carte

Date de validité Code crypto

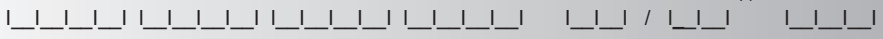

Date : ........ / .................

Signature 